\title{
Sífilis adquirida em Passo Fundo, Rio Grande do Sul, 2010 - 2017: incidência e distribuição da doença
}

\author{
Acquired Syphilis in Passo Fundo, Rio Grande do Sul, 2010 - 2017: incidence and disease \\ distribution \\ Sífilis adquirida en Passo Fundo, Rio Grande do Sul, 2010 - 2017: incidencia y distribución de la \\ enfermedad
}

Recebido: 25/01/2021 | Revisado: 26/01/2021 | Aceito: 30/01/2021 | Publicado: 07/02/2021
Deison Alencar Lucietto
ORCID: https://orcid.org/0000-0001-7002-7952 Universidade Federal Fluminense, Brasil E-mail: deisonlucietto@id.uff.br
Matheus Henrique Benin Lima ORCID: https://orcid.org/0000-0002-1609-1379 Universidade de Passo Fundo, Brasil E-mail: matheushbeninlima@gmail.com
Rodrigo Alberton da Silva
ORCID: https://orcid.org/0000-0003-2897-6606 Universidade de Passo Fundo, Brasil
E-mail: rodrigoalbertondasilva@gmail.com Ana Victória Coletto Reichert
ORCID: https://orcid.org/0000-0002-8487-6290 Universidade de Passo Fundo, Brasil E-mail: anavictoriareichert@gmail.com
Louise Pietrobon
ORCID: https://orcid.org/0000-0002-2383-1590 E-mail: pietrobonlouise@gmail.com
Daniela Bertol Graeff
ORCID: https://orcid.org/0000-0002-7182-8855 Universidade de Passo Fundo, Brasil E-mail: danibertol@upf.br Faculdade Especializada na Área da Saúde do Rio Grande do Sul, Brasil

\begin{abstract}
Resumo
Objetivo: descrever a incidência e a distribuição de casos de sífilis adquirida no município de Passo Fundo (Rio Grande do Sul) entre os anos de 2010 a 2017. Métodos: estudo transversal descritivo, realizado no Setor de Vigilância em Saúde da Secretaria Municipal da Saúde local. Foram incluídas as notificações disponibilizadas no SINAN. A análise ocorreu de forma descritiva, por meio da distribuição das notificações por variáveis sociodemográficas. Resultados: foram computadas 2.424 notificações de sífilis adquirida. Observou-se expressivo crescimento do número de notificações no decorrer do tempo. Ocorreram mais diagnósticos em homens ( $\mathrm{n}=1255 ; 51,8 \%)$, indivíduos de raça branca $(n=1997 ; 82,4 \%)$ e em indivíduos com ensino fundamental incompleto $(n=447 ; 18,4 \%)$ e com ensino médio completo $(n=584 ; 24,1 \%)$. Conclusão: esse estudo ressalta a importância de pesquisas nas regiões interioranas do país e a necessidade de novas investigações para melhor compreender os fatores relacionados ao grande número de casos de sífilis na cidade.
\end{abstract}

Palavras-chave: Sífilis; Incidência; Aplicações da Epidemiologia; Notificação de doenças.

\begin{abstract}
Objective: describe the incidence and distribution of acquired syphilis cases in Passo Fundo (Rio Grande do Sul) between 2010 and 2017. Methods: transversal descriptive study, realized in the Health Surveillance Sector of the Municipal Health Secretariat. Were included the notifications that were available on SINAN. The analysis occurred descriptively, with the SPSS program 18th version, through the notifications distribution in sociodemographic variables. Results: 2.424 acquired syphilis notifications were computed. An expressive growth in the notifications number was observed during the study time. Most of the diagnosis were in men $(51,8 \%)$, white people $(82,4 \%)$ and in persons with unfinished Elementary and Middle School (18,4\%) and with finished High School (24,1\%). Conclusion: this study highlights the importance of studies in the countryside of Brazil, the need of newer investigations so to understand better the factors related to the big number of syphilis cases in the city.
\end{abstract}


Keywords: Syphilis; Incidence; Uses of epidemiology; Disease notification.

\section{Resumen}

Objetivo: describir la incidencia y distribución de casos de sífilis adquirida en el municipio de Passo Fundo (Rio Grande do Sul) entre los años de 2010-2017. Método: estudio transversal descriptivo, realizado en el Sector de Vigilancia en Salude de la Secretaria Municipal de Salude. Fueran incluidas notificaciones disponibles en el SINAN. El análisis ocurrió de forma descriptiva, con el auxilio del Programa SPSS versión 18, mediante la distribución de las notificaciones por variables socio demográficas. Resultados: 2424 notificaciones fueron computadas. Se observó un crecimiento fuerte de los números en el transcurso del tiempo. Se han ocurrido más diagnósticos en hombres $(51,8 \%)$, individuos de raza blanca $(82,4 \%)$ y en individuos con enseñanza primaria incompleta $(18,4 \%)$ y secundaria completa $(24,1 \%)$. Conclusión: resaltase la importancia de búsquedas en interior del país y la necesidad de nuevas investigaciones a fin de comprender mejor los factores relativos a la gran cantidad de casos de sífilis en la ciudad.

Palabras clave: Sífilis; Incidencia; Usos de la epidemiología; Notificación de enfermedades.

\section{Introdução}

A sífilis, doença infectocontagiosa provocada pela espiroqueta Treponema pallidum (Rowley \& Hoorn, 2019), é transmitida principalmente por via sexual, a partir do contato direto com as lesões nas regiões genital e anal (sífilis adquirida) e através da transmissão vertical (binômio materno-fetal), a partir da via placentária ou hematogênica (sífilis congênita) (Gomes \& Meier, 2017). Apresenta evolução clínica de vários anos, variando desde quadros assintomáticos até o acometimento crítico de sistemas orgânicos (Sparling, 1990).

No Brasil, trata-se de uma patologia de notificação compulsória, devendo essa ser realizada obrigatoriamente por médicos, outros profissionais de saúde ou responsáveis pelos serviços públicos ou privados, diante de sua suspeita ou confirmação (Ministério da Saúde, 2016). Essas notificações integram o Sistema de Informação de Agravos e Notificações (SINAN), que assume função estratégica para a vigilância em saúde no âmbito do Sistema Único de Saúde (SUS).

De acordo com o Ministério da Saúde, existem diferentes formas de notificação do agravo: sífilis congênita (instituída em 1986), sífilis em gestantes (desde 2005) e sífilis adquirida (mais recente, incluída em 2010) (Ministério da Saúde, 2019). Os critérios de definição desses agravos foram redefinidos em 2017, pela Nota Informativa $N^{\circ} .2$ da Secretaria de Vigilância à Saúde do Ministério da Saúde (Ministério da Saúde, 2017).

Por se tratar de doença com métodos de diagnóstico simples e de baixo custo, possibilidade de tratamento há mais de meio século e não existir reservas em animais, possui características favoráveis para sua erradicação (Cavalcante \& Pereira, 2017). Entretanto, continua sendo um problema de saúde pública a nível mundial e estima-se que afete aproximadamente 17,7 milhões de indivíduos globalmente e que tenha uma incidência anual de 5,6 milhões de novos casos (Peeling \& Mabey, 2017).

No Brasil, as taxas de incidência de sífilis congênita, em gestantes e adquirida aumentaram significativamente nos últimos anos. Apenas no ano de 2018 foram notificados no SINAN 246.869 casos de sífilis, sendo 158.051 casos de sífilis adquirida, 62.599 casos de sífilis em gestantes e 26.219 casos de sífilis congênita.

No que se refere à sífilis adquirida, o Rio Grande do Sul teve 15.269 casos notificados em 2018, sendo o terceiro estado brasileiro com maior taxa de detecção da doença (134,8 casos/100.000 hab.) ficando atrás apenas de Santa Catarina (164,1 casos/100.000 hab.) e Mato Grosso do Sul (163,0 casos/100.000 hab.). Nesse ano, a média nacional foi de 75,8 casos/100.000 hab. (Ministério da Saúde, 2019).

Ante o exposto, este artigo tem como objetivo descrever a incidência e a distribuição de casos de sífilis adquirida no município de Passo Fundo/RS (referência em saúde no Rio Grande do Sul) entre os anos de 2010 a 2017, tendo como base de análise variáveis sociodemográficas e das unidades notificadoras.

\section{Metodologia}

Trata-se de estudo transversal descritivo de dados secundários, com abordagem quantitativa (Pereira \& Shitsuka, 
2018), realizado no Setor de Vigilância em Saúde da Secretaria Municipal da Saúde de Passo Fundo/RS (SMS/PF), cidade localizada na região Norte do Rio Grande do Sul. O município possui extensão territorial de 783,603 km² e população estimada de 203.275 habitantes (IBGE, 2019), sendo considerado importante polo de saúde na Região Sul do Brasil.

Foram incluídas as notificações de sífilis adquirida da localidade no período de 2010-2017 disponibilizadas no SINAN. É importante ressaltar que essa forma de sífilis está incluída como notificação compulsória desde 2010. Os casos que, a partir da Nota Informativa DIAHV/SVS/MS N. ${ }^{\circ}$ 2/2017 (Ministério de Saúde, 2016) passaram a ser classificados como sífilis em gestantes, foram considerados. Foram excluídas aquelas inelegíveis, inadequadamente preenchidas e de notificados não residentes em Passo Fundo/RS.

Foram levantadas as seguintes variáveis nas fichas de notificação: a) total de notificações: estratificação por ano no período 2010 a 2017; b) principais unidades notificadoras; e c) variáveis sociodemográficas dos casos: local de transmissão, idade, sexo, situação gestacional, raça, escolaridade e principais locais de residência.

Os dados foram analisados de forma descritiva, com o auxílio do programa estatístico disponível comercialmente Statistical Package for the Social Sciences (SPSS) versão 18.0 (SPSS Inc, Chicago, IL, USA), por meio da distribuição das notificações por variáveis sociodemográficas e da utilização de medidas prevalência e incidência da doença.

A pesquisa foi autorizada pela SMS/PF e aprovada, em suas questões éticas, via Plataforma Brasil, através do Registro CAAE Nº 83497318.5.0000.5342 e do Parecer Consubstanciado $N^{\circ}$ 2.627.373.

\section{Resultados}

Foram computadas 2.424 notificações de sífilis adquirida em Passo Fundo/RS entre os anos 2010 e 2017. Observou-se expressivo crescimento do número de notificações no decorrer do tempo. Sendo que em 2017, último ano incluído nessa análise, houve o maior número de notificações da doença no período (31,5\%) (Gráfico 1).

Gráfico 1 - Incidência de notificações de sífilis adquirida em Passo Fundo/RS no período 2010 a 2017 (n=2.424).

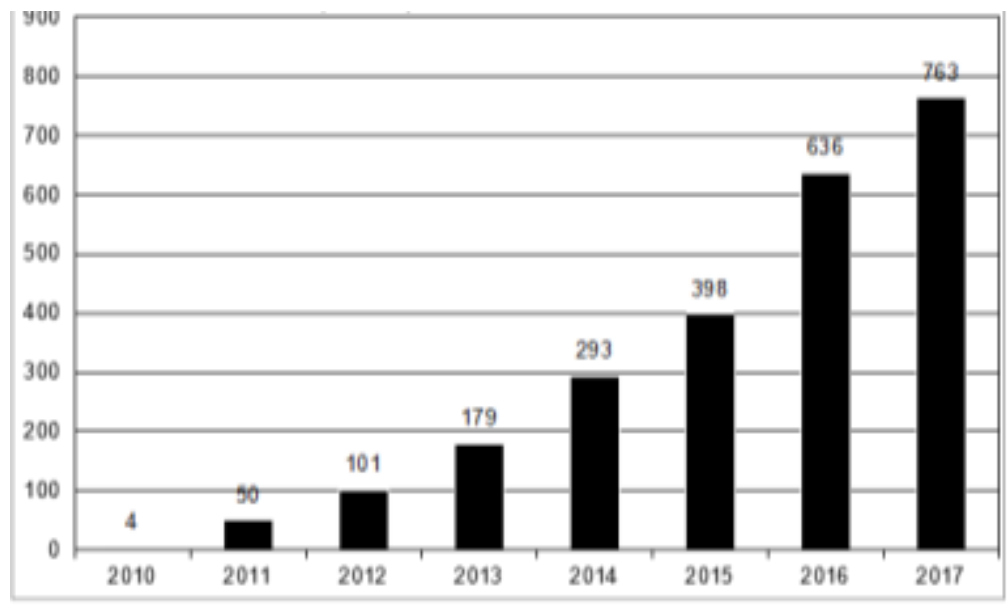

Fonte: SINAN/DATASUS.

Verificou-se que houve mais de 50 unidades notificadoras, sendo que cinco delas foram responsáveis por $69,0 \%$ das notificações de sífilis adquirida no município. Dessas principais unidades, houve destaque para hospitais e bancos de sangue (Tabela 1). 
Tabela 1 - Principais unidades notificadoras de sífilis adquirida em Passo Fundo/RS, no período de 2010 a $2017(n=2.424)$

\begin{tabular}{lcc}
\hline Unidade & n & $\%$ \\
Hospital São Vicente de Paulo & 569 & 23,5 \\
Hemopasso - Hemocentro Regional de Passo Fundo & 437 & 18,0 \\
Banco de Sangue Oswaldo Cruz & 348 & 7,4 \\
Serviço de Atendimento Especializado & 190 & 7,8 \\
Laboratório SANI & 128 & 5,3 \\
\hline
\end{tabular}

Fonte: SINAN/DATASUS.

Em relação à distribuição dos casos de sífilis adquirida, nas notificações com esse dado, constatou-se que houve predomínio de casos autóctones $(77,4 \%)$, ou seja, de pessoas que contraíram a doença na zona de sua residência. Dentre as notificações, 3,4\% foram consideradas não autóctones. Essa variável apresentou 276 casos ignorados e 190 perdidos (19,2\% do número total de notificações) (Tabela 2).

Tabela 2 - Distribuição dos casos de sífilis adquirida em Passo Fundo/RS no período de 2010 a 2017 segundo local de transmissão e variáveis sociodemográficas $(n=2.424)$.

\begin{tabular}{lcc}
\hline Local de transmissão & n & \% \\
\hline Casos autóctones & 1875 & 77,4 \\
Casos alóctone & 83 & 11,4 \\
Ignorado & 276 & 7,8 \\
Não preenchido & 190 & 51,8 \\
\hline Sexo & & 48,2 \\
\hline Masculino & 1255 & - \\
\hline Feminino & 1169 & 41,0 \\
\hline Ignorado & & - \\
\hline Não preenchido & 0 & 16 \\
\hline Gestantes & 0 & \\
\hline
\end{tabular}


Research, Society and Development, v. 10, n. 2, e12310212335, 2021

(CC BY 4.0) | ISSN 2525-3409 | DOI: http://dx.doi.org/10.33448/rsd-v10i2.12335

Segundo trimestre

15

38,4

Terceiro trimestre

6

15,3

Idade gestacional ignorada

2

5,1

Não grávida

Não se aplica

1543

Ignorado

129

Não preenchido

0

\section{Faixa etária}

\begin{tabular}{|c|c|c|}
\hline$<13$ anos & 5 & 0,2 \\
\hline 13-19 anos & 234 & 9,6 \\
\hline 20-29 anos & 829 & 34,2 \\
\hline 30-39 anos & 504 & 20,8 \\
\hline 40-49 anos & 353 & 14,6 \\
\hline$>50$ anos & 489 & 20,2 \\
\hline Ignorado & 0 & - \\
\hline Não preenchido & 10 & 0,4 \\
\hline \multicolumn{3}{|l|}{ Raça/cor da pele } \\
\hline Branca & 1997 & 82,4 \\
\hline Preta & 87 & 3,6 \\
\hline Amarela & 8 & 0,3 \\
\hline Parda & 208 & 8,6 \\
\hline Indígena & 9 & 0,3 \\
\hline Ignorado & 103 & 4,2 \\
\hline Não preenchido & 12 & 0,5 \\
\hline
\end{tabular}

Escolaridade 
Research, Society and Development, v. 10, n. 2, e12310212335, 2021

(CC BY 4.0) | ISSN 2525-3409 | DOI: http://dx.doi.org/10.33448/rsd-v10i2.12335

\begin{tabular}{|c|c|c|}
\hline $1^{\mathrm{a}}-4^{\mathrm{a}}$ série incompleta do $\mathrm{EF}$ & 87 & 3,6 \\
\hline $4^{\mathrm{a}}$ série completa do $\mathrm{EF}$ & 35 & 1,4 \\
\hline $5^{\mathrm{a}}-8^{\mathrm{a}}$ série incompleta do Ensino Fundamental & 447 & 18,4 \\
\hline Ensino Fundamental Completo & 280 & 11,6 \\
\hline Ensino Médio Incompleto & 187 & 7,7 \\
\hline Ensino Médio Completo & 584 & 24,1 \\
\hline Ensino Superior Incompleto & 132 & 5,4 \\
\hline Ensino Superior Completo & 122 & 5,0 \\
\hline Ignorado & 445 & 18,4 \\
\hline Não preenchido & 14 & 0,6 \\
\hline \multicolumn{3}{|l|}{ Bairro de residência } \\
\hline Centro & 252 & 10,4 \\
\hline Vera Cruz & 203 & 8,4 \\
\hline Petrópolis & 164 & 6,8 \\
\hline São José & 117 & 4,8 \\
\hline São Luiz Gonzaga & 117 & 4,8 \\
\hline Outros & 1548 & 63,9 \\
\hline Não preenchido ou ignorado & 24 & 1,0 \\
\hline
\end{tabular}

Fonte: SINAN/DATASUS.

Houve pequena diferença em relação ao sexo, com maior predomínio para homens (51,8\%). Dentre os 2.424 casos analisados, 39 mulheres eram gestantes $(1,6 \%)$. Quanto à idade gestacional, 41,0\% estavam no $1^{\circ}$ trimestre. No que se refere à idade, foram notificados casos de 1 até 91 anos de idade, sendo a média etária de 35,4 anos. Cerca de um terço dos indivíduos encontravam-se entre 20-29 anos (Gráfico 2 e Tabela 2). 
Gráfico 2 - Distribuição dos casos de sífilis adquirida em Passo Fundo/RS por faixa etária, no período 2010 a 2017 $(n=2.424)$.

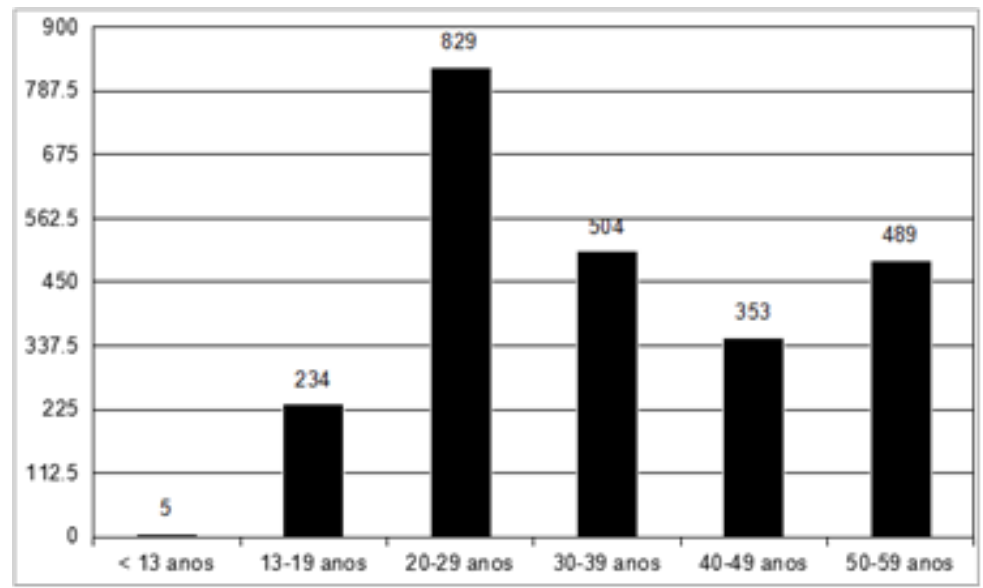

Fonte: SINAN/DATASUS.

Identificou-se mais diagnósticos em indivíduos de raça branca (82,4\%). Quanto à escolaridade, 18,4\% dos portadores declaram escolaridade como Ensino Fundamental Incompleto ( $5^{\mathrm{a}}-8^{\mathrm{a}}$ série) e $24,1 \%$ como Ensino Médio Completo (Gráfico 3). Por fim, quanto à localização residencial, identificaram-se mais de 100 localidades. Os cinco bairros mais citados foram: Centro (10,4\%); Vera Cruz (8,4\%); Petrópolis (6,8\%); São José (4,8\%); e São Luiz Gonzaga (4,8\%) (Tabela 2).

Gráfico 3 - Distribuição dos casos de sífilis adquirida em Passo Fundo/RS por grau de escolaridade, no período 2010 a 2017 $(\mathrm{n}=2.424)$.

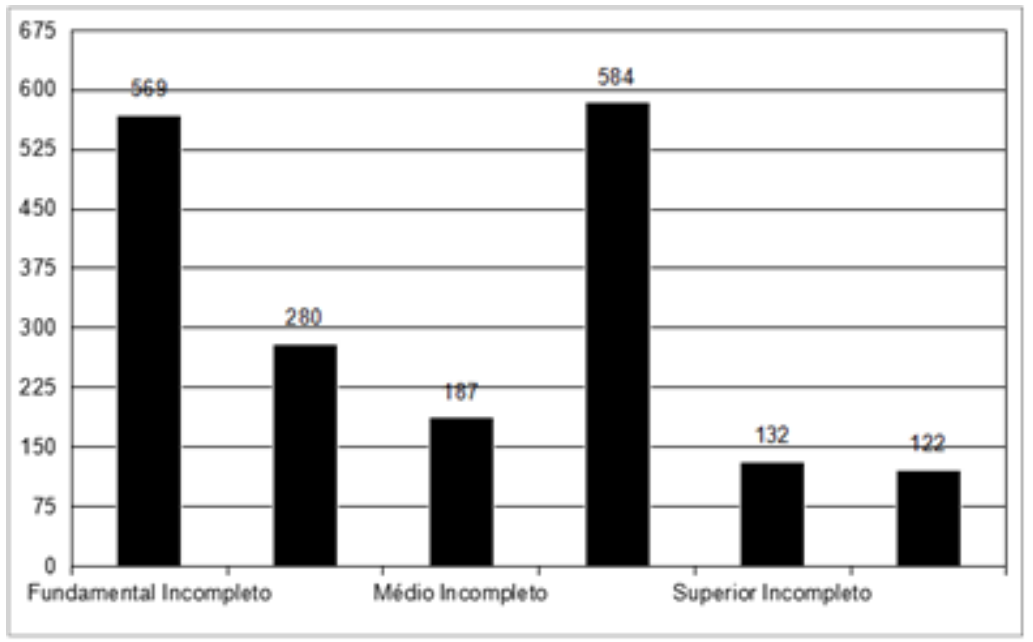

Fonte: SINAN/DATASUS.

\section{Discussão}

No Brasil, durante o período de 2010 a junho de 2017, dados do SINAN apontaram para 342.531 casos de sífilis adquirida (Ministério da Saúde, 2017). Em 2017, o país teve 119.800 casos, sendo que a taxa de detecção de sífilis adquirida foi de 58,1 casos/100 mil habitantes (Ministério da Saúde, 2018). Esses dados identificam uma alta incidência no país, apresentando valores acima de países como Estados Unidos, Austrália, Eslováquia, Reino Unido e outros países europeus (Mattei \& Beachkofsky, 2012).

Sabe-se, nesse sentido, que a sífilis se apresenta especialmente em países de baixa e média renda (Rowley, et al., 
2019). Nos países desenvolvidos, sua presença se dá majoritariamente em populações vulneráveis, caracterizadas por pobreza/limitação econômica, pouco acesso ao sistema de saúde e minorias étnicas e sexuais (CDC, 2015).

Dos casos notificados de sífilis adquirida entre 2010 e 2017, 21,2\% (72.616 casos) ocorreram na região Sul (Ministério da Saúde, 2017). No ano de 2017, especificamente, a Região Sul contabilizou 29.169 casos, correspondendo a $24,3 \%$ do país. Nesse ano, o Estado do Rio Grande do Sul teve 13.114 casos (10,9\% em relação ao país e 45,0\% em relação à Região Sul). Desses, 2.424 foram notificados em Passo Fundo/RS, o que corresponde a 18,5\% dos casos estaduais (Ministério da Saúde, 2018).

O Estado do Rio Grande do Sul teve a segunda maior taxa de detecção de sífilis adquirida do país no ano de 2017, com 116,2 casos/100 mil habitantes. Nesse mesmo ano, o munícipio de Passo Fundo/RS apresentou 381,5 casos/100 mil habitantes, taxa 3,2 vezes maior que a estadual e 6,5 vezes maior que a nacional (51,8 casos/100 mil habitantes) (Ministério da Saúde, 2018). Dessa forma, os dados apresentados pelo presente trabalho identificaram diferenças importantes entre os dados epidemiológicos da cidade em relação aos dados estaduais e nacionais. Acredita-se que dentre os fatores que possam elucidar essa diferença estão o acesso da população local aos serviços diagnósticos e as características da população local, com forte presença de jovens universitários, tendo essas hipóteses a necessidade de maior investigação.

De 2010 a 2017, a taxa de detecção de sífilis em Passo Fundo/RS apresentou um crescimento anual importante (Gráfico 1). Fato semelhante foi identificado entre os dados nacionais, quando a taxa de detecção saltou de 2,0 para 58,1 casos a cada 100.000 habitantes (Ministério da Saúde, 2018).

Esse comportamento da doença tem sido denominado, nas últimas décadas, de epidemia ou ressurgimento da sífilis (Arando \& Caballero, 2019; Arora \& Schuenemann, 2016). Após a incidência de sífilis ter diminuído na década de 1940, com a introdução da penicilina, observaram-se novos crescimentos e surtos a partir dos anos 1980 e dos anos 2000 (Mattei, et al., 2012; Arora, et al., 2016; Kitayama \& Segura, 2017; O'Byrne \& MacPherson, 2019; Klausner, 2019).

Grande parte dos países ocidentais, inclusive países desenvolvidos, apresentaram aumento nos números de sífilis desde os anos 2000, chegando a 300\% (Arora, et al., 2016; O'Byrne, et al., 2019). Em 2016, a prevalência e a incidência global foram de 0,5\% e 1,6 a 1,7 casos/1000 pessoas, correspondendo a 6 milhões de infectados no ano e 19,9 milhões de infectados no total (Rowley et al., 2019).

Dentre os possíveis motivos ligados a essa nova epidemia de sífilis, destaca-se o retorno de hábitos sexuais vulneráveis, os quais parecem estar ligados à diminuição do medo do vírus da imunodeficiência humana (VIH) e à redução da mortalidade da infecção pelo HIV, com o surgimento de novos antirretrovirais (Klausner, 2019; Kojima \& Klausner, 2018; Luppi \& Gomes, 2018; Nunes \& Zara, 2018). No fim do século XX, o impacto do VIH foi responsável por uma diminuição transitória da incidência de sífilis (e outras ISTs) (Klausner, 2019).

Além disso, acredita-se que a redução no uso do preservativo masculino, o aumento dos casos entre os homens que fazem sexo com homens (e outras populações de risco) (Klausner, 2019; Kojima, et al., 2018; Luppi, et al., 2018; Barbosa \& Batista, 2019) e a diminuição dos serviços públicos de prevenção (Klausner, 2019) possam explicar o fenômeno do ressurgimento dessa doença nos últimos anos em nível global.

No Brasil, além desses fatores, supõe-se que o aumento do número de casos pode estar relacionado a questões ligadas ao sistema de saúde, em função dos avanços na atenção primária em saúde e Estratégia de Saúde da Família, incluído a descentralização dos serviços e a maior acessibilidade de testes rápidos para o seu diagnóstico (Nunes, et al., 2018; Silva \& Barreto, 2020). Apesar disso, chamou a atenção que 69,0\% das notificações de sífilis adquirida em Passo Fundo/RS foram realizadas por cinco unidades notificadoras, com destaque para hospitais e bancos de sangue.

Nessa pesquisa identificou-se pequena diferença percentual entre os sexos nos casos notificados entre 2010 e 2017 , com maior participação de homens $(51,8 \%)$, sendo esses dados divergentes em relação ao comportamento da doença em nível 
nacional e internacional. No Brasil, em 2017, 58,4\% dos casos de sífilis adquirida eram em mulheres (Ministério da Saúde, 2018). Em 2015, dados do Centers for Disease Control and Prevention (CDC) sugerem uma diferença ainda maior, quando a taxa de casos de sífilis entre homens foi 7 vezes maior que entre mulheres (CDC, 2015). Estudo realizado em Barcelona identificou 97,0\% de casos em indivíduos do sexo masculino (Arando, et al., 2019).

Além disso, ainda em relação ao sexo, verificou-se pequena participação de notificações de casos em gestantes na pesquisa $(1,6 \%)$, o que pode ser explicado pelas mudanças provocadas a partir da Nota Informativa DIAHV/SVS/MS N. ${ }^{\circ}$ 2/2017 (Ministério da Saúde, 2017).

Observou-se, neste estudo, que 34,2\% dos casos notificados situavam-se entre 20 e 29 anos, identificando predomínio de adultos jovens. Esse achado se assemelha aos dados nacionais de 2017, quando a maior parte das notificações de sífilis adquirida ocorreu em indivíduos da mesma faixa etária (35,3\%) (Ministério da Saúde, 2018) e a um estudo paulista que encontrou cerca de 50\% dos portadores possuía entre 25 e 34 anos (Luppi, et al., 2018). A idade média referida pelo estudo barcelonês foi de 35,3 anos (Arando, 2019), semelhante ao encontrado nesse trabalho.

Contudo, identificou-se nessa série histórica que 20,2\% dos casos se encontraram na faixa etária acima de 50 anos, sendo o terceiro maior grupo no número de incidência na população de Passo Fundo/RS. Isto pode ser analisado pela tendência na redução da utilização de preservativos conforme o aumento da idade (Lazzarotto \& Kramer, 2008), aspecto influenciado pela falta de preocupação com a possiblidade de uma gestação.

A partir desses achados, pode-se inferir que as estratégias de prevenção e deteç̧ão devem abranger, de modo prioritário, os adultos jovens. Por outro lado, é importante ressaltar que a sífilis pode ser uma hipótese diagnóstica em qualquer faixa etária.

Em relação à raça/cor declarada, houve uma grande diferença percentual entre os dados encontrados em Passo Fundo/RS e os nacionais. A proporção de brancos foi muito maior que as de outras raças, alcançando 82,4\%. Destaca-se uma baixa quantidade de notificações assinaladas como pardas na cidade $(8,6 \%)$. Dentre todas as notificações nacionais, no ano de 2017, 38,8\% dos casos declararam-se brancos, 32,9\% pardos, 9,4\% pretos e 0,5\% indígenas (Ministério da Saúde, 2018).

Nesse sentido, é preciso pontuar que a cidade de Passo Fundo/RS apresenta colonização predominantemente italiana e alemã. De acordo com o censo demográfico realizado pelo IBGE, em 2010, 47,5\% da população brasileira se classificava como branca, $43,1 \%$ como parda e 7,6\% como preta, enquanto em Passo Fundo/RS esse percentual era, respectivamente de $83,2 \%, 13,9 \%$ e $2,5 \%$ (IBGE, 2019).

No que se refere à escolaridade, verificou-se que 23,5\% dos indivíduos desse estudo apresentavam ensino fundamental incompleto e 24,1\% possuíam ensino médio completo, no período analisado. Dados do Brasil para o ano de 2017 demonstram que percentual menor, de $19,4 \%$ e $18,1 \%$, respectivamente. Entretanto, houve elevado percentual de notificações em que a informação sobre escolaridade foi preenchida como "ignorada" ou não houve preenchimento do campo, sendo de 18,9\% entre 2010-2017 em Passo Fundo/RS e 26,1\% em 2017 no Brasil (Ministério da Saúde, 2018).

Apesar do grande percentual de campos preenchidos como ignorado, e considerando a escolaridade um reflexo da condição socioeconômica, é possível visualizar grande distribuição da doença entre diferentes classes sociais, o que também pressupõe a proposição de diversificadas estratégias de enfrentamento na localidade.

Quanto à localização residencial, identificou-se, nesse estudo, mais de 100 localidades nas fichas de notificação, sugerindo ampla distribuição da doença nos bairros do município de Passo Fundo/RS. Dentre os cinco bairros mais citados estão aqueles com maior concentração populacional, em virtude da presença de comércios e serviços, inclusive educacionais, na cidade.

Acredita-se que a redução da subnotificação de sífilis adquirida, em função da sua notificação compulsória (desde 2010) seja importante fator a ser considerado para o seu manejo (Luppi, et al., 2018), uma vez que a perda de seguimento de 
casos e tratamento inadequado dos indivíduos ainda figuram como importantes problemáticas para o seu controle no país (Nunes, et al., 2018).

Devido às diferenças culturais e comportamentais, sabe-se que estudos epidemiológicos de alcance nacional não refletem particularidades de cada local (Barbosa, et al., 2019), sendo necessário o desenvolvimento de trabalhos a nível regional e local, mesmo diante de limitações oriundas de falhas no preenchimento das fichas de notificação, como o proposto nesse estudo.

Por isso, reforça-se que a realização de pesquisas como essa, fundamentada em informações disponibilizadas no SINAN, é de grande relevância para conhecer o comportamento e a distribuição da doença em cada localidade/região brasileira, possibilitando a realização de diagnósticos, o fornecimento de explicações e a indicação de riscos, favorecendo a tomada de decisão com vistas ao planejamento e avaliação em saúde pública.

\section{Considerações Finais}

A análise da incidência e da distribuição de sífilis adquirida através das notificações de doença em Passo Fundo/RS no período 2010-2017 revelou elevado número de casos e incidência anual crescente, quando as taxas de detecção foram bastante superiores às médias estadual e nacional. Houve predomínio de casos autóctones, em indivíduos do sexo masculino, brancos, entre 20-29 anos, com ensino fundamental incompleto e moradores de diferentes bairros da cidade. Verificou-se, também, que as principais unidades notificadoras do município foram serviços hospitalares e laboratoriais.

Diante do fenômeno do ressurgimento da epidemia de sífilis nas últimas décadas, bem como de suas repercussões sistêmicas, as quais são acompanhadas de sofrimento, preconceitos e exclusão social, esse estudo ressalta a importância de pesquisas nas regiões interioranas do país, a necessidade de novas investigação para melhor compreender os fatores relacionados ao grande número de casos de sífilis na cidade e, também, a proposição de medidas urgentes para a prevenção e controle dessa doença na população local.

Tendo em mente os achados dessa pesquisa, sugere-se a realização de estudos que analisem dados complementares da ficha de notificação, como ocupação, antecedente de sífilis, realização de tratamento e comportamento sexual, bem como a condução de pesquisas com metodologia qualitativa, de modo a compreender o nível de informação, as práticas, opiniões, crenças e vivências de pessoas com diagnóstico de sífilis adquirida, bem como o itinerário terapêutico dessas pessoas no sistema de saúde, desde a busca por atendimento até a finalização do tratamento.

\section{Referências}

Arando, M., Caballero, E., Curran, A., Armengol, P., Barberá, M. J., \& Vall-Mayans, M. (2019). The Epidemiological and Clinical Characteristics of the Epidemic of Syphilis in Barcelona. Actas Dermosifiliograficas, 110(10), 841-849. 10.1016/j.ad.2019.03.014.

Arora, N., Schuenemann, V. J., Jäger, G., Peltzer, A., Seitz, A., Herbig, A., et al (2016). Origin of modern syphilis and emergence of a pandemic Treponema pallidum cluster. Nature Microbiology, 2:16245. 10.1038/nmicrobiol.2016.245

Barbosa, K. F., Batista, A. P., Nacife, M. B., Vianna, V. N., Oliveira, W. W., Machado, E. L., et al (2019). Fatores associados ao não uso de preservativo e prevalência de HIV, hepatites virais B e C e sífilis: estudo transversal em comunidades rurais de Ouro Preto, Minas Gerais, entre 2014 e 2016. Epidemiologia e. Serviços de Saúde, 28(2) http://dx.doi.org/10.5123/s1679-49742019000200023.

Cavalcante, P. A. M., Pereira, R. B.L., Castro, J. G. D. (2017). Sífilis gestacional e congênita em Palmas, Tocantins, 2007-2014. Epidemiologia e Serviços de Saúde; 26(2): 255-264. 10.5123/S1679-49742017000200003

Centers for Disease Control and Prevention (EUA). Division of STD Prevention (2015). Sexually Transmitted Disease Surveillance 2014. Atlanta: Centers for Disease Control and Prevention.

Centers for Disease Control and Prevention (EUA) (2015). Sexually transmitted diseases treatment guidelines. Atlanta: Centers for Disease Control and Prevention

Gomes, N. C. R. C., Meier, D. A. P., Pieri, F. M., Alves, E., Albanese, S. P. R., Lentine, E. C., et al (2017). Prevalence and factors associated with syphilis in a Reference Center. Revista da Sociedade Brasileira de Medicina Tropical, 50(1): 27-34. http://dx.doi.org/10.1590/0037-8682-0102-2016 
Instituto Brasileiro de Geografia E Estatística (IBGE) (2019). Estimativa Populacional de Passo Fundo/RS - 2019 [Internet]. Rio de Janeiro: Instituto Brasileiro de Geografia e Estatística.

Kitayama, K., Segura, E. R., Lake, J. E., Perez-Brumer, A. G., Oldenburg, C. E., Myers, B. A., et al (2017). Syphilis in the Americas: a protocol for a systematic review of syphilis prevalence and incidence in four high-risk groups, 1980-2016. Systematic Reviews, 6:195 10.1186/s13643-017-0595-3

Klausner, J. D. (2019). The great imitator revealed: syphilis. Topics in Antiviriral Medicine., 27(2):71-74.

Kojima, N., \& Klausner, J. D. (2018). An Update on the Global Epidemiology of Syphilis. Current Epidemiology Reports, 5(1):24-38. 10.1007/s40471-0180138-z.

Lazzarotto, A. R., Kramer, A. S., Hädrich, M., Tonin, M., Caputo, P., \& Sprinz, E. (2008). O conhecimento de HIV/aids na terceira idade: estudo epidemiológico no Vale do Sinos, Rio Grande do Sul, Brasil. Ciência e Saúde Coletiva, 13(6): http://dx.doi.org/10.1590/S1413-81232008000600018.

Luppi, C. G., Gomes, S. E., Silva, R. J., Ueno, A. M., Santos, A. M., Tayra, A., et al (2018). Fatores associados à coinfecção por HIV em casos de sífilis adquirida notificados em um Centro de Referência de Doenças Sexualmente Transmissíveis e Aids no município de São Paulo, 2014. Epidemiologia e Serviços de Saúde, 27(1) http://dx.doi.org/10.5123/s1679-49742018000100008.

Mattei, P. L., Beachkofsky, T. M., Gilson, R. T., \& Wisco, O. J. (2012). Syphilis: a reemerging infection. American Family Physician. 1;86(5), 433-40

Pereira, A. S., Shitsuka, D. M., Parreira, F. B., \& Shitsuka. R (2018). Metodologia da pesquisa científica, UFSM, NTE.

Ministério da Saúde (BR). Secretaria de Vigilância em Saúde (2017). Boletim Epidemiológico de Sífilis. Brasília: Ministério da Saúde.

Ministério da Saúde (BR). Secretaria de Vigilância em Saúde (2018). Boletim Epidemiológico de Sífilis. Brasília: Ministério da Saúde.

Ministério da Saúde (BR). Secretaria de Vigilância em Saúde (2019). Sífilis 2019: Boletim Epidemiológico Especial. Brasília: Ministério da Saúde.

Ministério da Saúde (BR) (2016). Portaria N 204, de 17 de fevereiro de 2016. Define a Lista Nacional de Notificação Compulsória de doenças, agravos e eventos de saúde pública nos serviços de saúde públicos e privados em todo o território nacional, nos termos do anexo, e dá outras providências [Internet]. Diário Oficial da União, Brasília (DF).

Ministério da Saúde (BR). Secretaria de Vigilância à Saúde (2017). Nota Informativa n n 2-SEI/2017-DIAHV/SVS/MS, de 19 de setembro de 2017. Altera os Critérios de Definição de Casos para notificação de Sífilis Adquirida, Sífilis em Gestantes e Sífilis Congênita [Internet]. Ministério da Saúde, Brasília (DF)

Nunes, P. S., Zara, A. L., Rocha, D. F., Marinho, T. A., Mandacarú, P. M., \& Turchi, M. D. (2018). Sífilis gestacional e congênita e sua relação com a cobertura da Estratégia Saúde da Família, Goiás, 2007-2014: um estudo ecológico. Epidemiologia e Serviços de Saúde, 27(4). http://dx.doi.org/10.5123/s1679-49742018000400008.

O'Byrne, P., \& MacPherson, P. S. (2019). British Medical Journal, 365:14159. 10.1136/bmj.14159. Erratum in: BMJ. 19; $366: 14746$.

Peeling, R. W., Mabey, D., Kamb, M. L., Chen, X. S., Radolf, J. D., \& Benzaken, A. S. (2017). Syphilis. Nature Review Disease Primers, 3:17073. 10.1038/nrdp.2017.73.

Rowley, J., Vander Hoorn, S., Korenromp, E., Low, N., Unemo, M., Abu-Raddad, L. J., Chico, R. M., et al (2016). Chlamydia, gonorrhoea, trichomoniasis and syphilis: global prevalence and incidence estimates. Bulletin of the World Health Organization, 97(8), 548-562.

Silva, M. J., Barreto, F. R., Costa, M. C., Carvalho, M. S., \& Teixeira, M. G. (2020). Congenital syphilis distribution in the State of Tocantins, Brazil, $2007-$ 2015. Epidemiologia e Serviços de Saúde, 29 (2) http://dx.doi.org/10.5123/s1679-49742020000200017.

Sparling, P. F. (1990). Natural history of syphilis. In: Holmes, K. K., Mardh, P. A., Sparling, P. F., et al (Eds). Sexually Transmitted Diseases. McGraw-Hill, New York 1990. p.213. 